\title{
Solenoid Driven Pressure Valve System: Toward Versatile Fluidic Control in Paper Microfluidics
}

Taehoon H. Kim, Young Ki Hahn, J ungmin Lee, Danny van Noort and Minseok S. Kim

The self-archived postprint version of this journal article is available at Linköping University Institutional Repository (DiVA):

http:// urn.kb.se/ resolve?urn=urn:nbn:se:liu:diva-145759

N.B.: When citing this work, cite the original publication.

Kim, T. H., Hahn, Y. Ki, Lee, J., van Noort, D., Kim, M. S., (2018), Solenoid Driven Pressure Valve System: Toward Versatile Fluidic Control in Paper Microfluidics, Analytical Chemistry, 90(4), 25342541. https:// doi.org/ 10.1021/acs.analchem.7b03791

Original publication available at:

https:// doi.org/ 10.1021/ acs.analchem.7b03791

Copyright: American Chemical Society

http:// pubs.acs.org/ 


\title{
Solenoid Driven Pressure Valve System: Toward Versatile Fluidic Control in Paper Microfluidics
}

\author{
Taehoon H. Kim, ${ }^{\dagger, \perp}$ Young Ki Hahn, ${ }^{\ddagger, \perp}$ Jungmin Lee, ${ }^{\dagger}$ Danny van Noort, ${ }^{\dagger, \S}$ and Minseok S. Kim*, ${ }^{*}, \|_{\odot}$ \\ ${ }^{\dagger}$ Department of New Biology, Daegu Gyeongbuk Institute of Science and Technology (DGIST), Daegu 42988, Republic of Korea \\ ${ }^{*}$ Samsung Electronics, 4 Seocho-daero 74-gil, Seocho-gu, Seoul 06620, Republic of Korea \\ ${ }^{\S}$ Division of Biotechnology, Department of Physics, Chemistry and Biology (IFM), Linköping University, Linköping 58183, Sweden \\ "Well-Aging Research Center, Daegu Gyeongbuk Institute of Science and Technology (DGIST), Daegu 42988, Republic of Korea
}

Supporting Information

ABSTRACT: As paper-based diagnostics has become predominantly driven by more advanced microfluidic technology, many of the research efforts are still focused on developing reliable and versatile fluidic control devices, apart from improving sensitivity and reproducibility. In this work, we introduce a novel and robust paper fluidic control system enabling versatile fluidic control. The system comprises a linear push-pull solenoid and an Arduino Uno microcontroller. The precisely controlled pressure exerted on the paper stops the flow. We first determined the stroke distance of the solenoid to obtain a constant pressure while examining the fluidic time delay as a function of the pressure. Results showed that strips of grade 1 chromatography paper had superior reproducibility in fluid transport. Next, we characterized the reproducibility of the fluidic velocity which depends on the type and grade of paper used. As such, we were able to control the flow velocity on the paper and also achieve a complete stop of flow with a pressure over $2.0 \mathrm{MPa}$. Notably, after the actuation of the pressure driven valve (PDV), the previously pressed area regained its original flow properties. This means that, even on a previously pressed area, multiple valve operations can be successfully conducted. To the best of our knowledge, this is the first demonstration of an active and repetitive valve operation in paper microfluidics. As a proof of concept, we have chosen to perform a multistep detection system in the form of an enzyme-linked immunosorbent assay with mouse IgG as the target analyte.
$\mathrm{M}$ icrofluidic paper-based analytical devices ( $\mu$ PADs) have been extensively studied due to their unique advantages over conventional diagnostic systems, which include cost effectiveness, easy production, ease of use, reagent storage, biocompatible flexible substrate with high porosity, and powerfree fluid transport via capillary actuation. ${ }^{1,2}$ With these vast benefits, a variety of fabrication approaches (e.g., inkjet printing, ${ }^{3}$ photolithography, ${ }^{4}$ stamping, ${ }^{5}$ and embossing $\left.{ }^{6}\right)$ and capabilities (e.g., fluidic timers, ${ }^{7}$ metering, ${ }^{8}$ thermochromic displays, ${ }^{9}$ and text display ${ }^{10}$ ) have been developed and applied to the analysis of food and beverage contamination and environmental and biomedical analytes. ${ }^{11-14}$ Analytical assays can be classified in a single step and multistep. Most commercial products are based on the former (e.g., glucose, ${ }^{15}$ lactate, ${ }^{16}$ and uric acid assay ${ }^{17}$ ) and are, from reagent reaction to detection, a one-time operation, once a sample is loaded. Multistep assays, on the other hand, require several sequential reaction steps between sample introduction and detection. Such assays have been demonstrated to improve diagnostic performance, thereby enabling more diverse biochemical analyses, such as enzyme-linked immunosorbent assay (ELISA). ${ }^{18}$ Although this method improves the sensitivity, with every step, it requires manual manipulation to load the reagents. ${ }^{18-20}$

To overcome these limitations, there have been several studies done to minimize the manual steps by developing paper-based fluidic valves. ${ }^{21-30}$ These valves can be classified as either passive or active, according to the operation principle. While passive valve systems generally operate within autonomous structures, ${ }^{31}$ the specific geometry of the channel, ${ }^{24}$ or modified surface chemistry, ${ }^{21}$ active valve systems are actuated by mechanical device or external energy, ${ }^{25,32}$ making valve control more programmable. Notable passive valves were developed by Toley et al. ${ }^{33}$ and Chen et al. ${ }^{21}$ The former valve created a toolkit of paper microfluidic valves using movable paper strips and fluid-triggered expanding elements. The system comprises a new strategy of multiple functional valve and calculates automatic valve actuation although it requires a relatively complicated design because of the necessity of

Received: September 16, 2017

Accepted: January 24, 2018

Published: January 24, 2018 
actuation channels and only works once. The latter valve, a "fluidic diode", relies on patterning wettability on paper by click chemistry. The system is a two-terminal component that allows wicking in one direction but stops it in the other direction. However, surfactants used in this system may affect denaturalization of proteins. Another passive valve utilizes a dissolvable bridge as an off switch. The advantage of this valve is that it is a simple design to manipulate fluid transport but is a one-time operation and has limited fluid volume. ${ }^{34}$ Solventdependent on/off valves were introduced by utilizing alkyl ketene dimer patterning on paper. ${ }^{28}$ This approach enables selectively permeable on/off valves over only off switches. But the use of alcohol might affect interaction with sample reagents and limit their uses only to specific applications.

Given that studies for paper fluidic active valves have not yet been extensively developed, compared to passive valves, they have exhibited relatively simple paper channel geometry and fast actuation time. Simple, but powerful, active valves were introduced, among others, thermally actuated wax-ink valves printed onto the paper or electrical modification of wetting property by conductive hydrophilic and hydrophobic electrode/valves. $^{32,35}$ While there are many advantages of repeatable valve actuation, including ease of fabrication and system construction, care should be taken when dealing with temperature-sensitive samples and hydrophobic materials which could react with wax. Therefore, it is critically important to develop a novel paper-based fluidic active valve system which satisfies repeatable, instant, and programmable valve operations; does not exhibit sample variation due to valve components; and shows no occurrence of reactions between sample/biochemical reagents and any materials required for the valve function.

In this study, we developed a robust and bifunctional mechanical system for flow velocity control and valve actuation. Many studies have demonstrated either a delay in flow or an imperfect flow obstruction by a valve. In contrast, the solenoid pressure driven valve (PDV) in our system enables not only flow control but also a complete valve actuation function. Since the bifunctional valve is operated solely by mechanical pressure, fluidic control can be performed under normal conditions, which means that the paper itself does not require any additional modifications with materials which are either organic, hydrophobic, and/or hydrophilic. In addition, it does not need complicated and long paper channels, compared to the $\mu \mathrm{PADs}$ using passive valves. ${ }^{21}$ These latter devices require larger amounts of sample and reagents because of the longer channel length and, therefore, higher evaporation loss of the fluidic reagents.

We constructed our PDV system using an Arduino microprocessor to potentially enable programmable fluidic control on a mobile platform. The flow was evaluated as a function of the type and grade of paper, paper width, and method of channel fabrication, in order to achieve optimal reproducible conditions. Afterward, we examined the fluidic time delay according to the applied pressure on the valve and confirmed the possibility of flow velocity control. Notably, complete valve actuation was achieved when a pressure over 2 $\mathrm{MPa}$ was applied. To illustrate the effectiveness of our PDV system, we performed an HRP-based ELISA. Having the access to repeatable actuation means that we are able to construct a more reliable paper-based in vitro diagnostics tool for more complex immunoassays, including simplified multistep washing procedures and reagents.

\section{EXPERIMENTAL SECTION}

Preparation of Paper Substrates. Bare paper strips with widths of 2, 4, 6, 8, and $10 \mathrm{~mm}$ were cut from chromatography paper (grade 1, GE Healthcare Whatman, Springfield Mill, U.K.) and filter papers (grade 1, GE Healthcare Whatman) using a craft knife. Hydrophilic channels with 2, 4, 6, 8, and 10 $\mathrm{mm}$ widths were patterned on each piece of chromatography and filter paper using a wax printer (ColorQube 8570, Xerox, CT, USA). We printed wax patterns of 2.6, 4.6, 6.6, 8.6, and $10.6 \mathrm{~mm}$ widths onto cut paper, after which hydrophilic channels were formed by baking the wax-printed papers on a hot plate (HS-7, IKA, Guangzhou, China) at $150{ }^{\circ} \mathrm{C}$ for $1 \mathrm{~min}$.

PDV System. A support was designed by SolidWorks (Dassault Systemes SE, Velizy-Villacoublay, France) to attach to a linear solenoid (ZHO-1364S-36A13, Shenzhen Zonhen Electric Appliances, Shenzhen, China; $4 \mathrm{~cm} \times 3 \mathrm{~cm} \times 10 \mathrm{~cm}$ ) and was fabricated using a 3D printer (LM300, K.Clone, South Korea) with polylactic acid (PLA) filaments $(1.75 \mathrm{~mm}$, Esun, Shenzen, China). The linear solenoid and fabricated support were put together with screws $(2.5 \mathrm{~mm}$ diameter).

We used a load cell (TAS-606, HT sensor technology, Xian, China) fixed to the support below the linear solenoid to measure the mechanical force of the latter. When a current was applied to the linear solenoid from a power supply (RDP-303 AU, GEST Co., South Korea), the plunger struck the sensor of the load cell. The mechanical force of the linear solenoid was measured by a strain gauge sensor connected to the load cell amplifier (HX711, AVIA semiconductor, Xiamen, China) at the center of the load cell. Amplified signals were then digitalized by a microprocessor (Arduino UNO, Arduino Co., Turin, Italy) and displayed on an LCD screen (LCM1602 IIC V1, YwRobot, Shenzhen, China) connected to the microprocessor.

The surfaces of the fabricated support and the plunger head of the linear solenoid actuator were not smooth, meaning that the apparatus might incur low reproducibility and effectiveness when controlling fluids. To improve the performance, we fabricated a poly(dimethylsiloxane) (PDMS; Sylgard 184, Dow Corning, MI, USA) cap to cover the plunger head by placing the plunger head in a mold containing a mixture of PDMS base and curing agent (Sylgard 184, Dow Corning; 10:1 ratio), after which the PDMS was cured in a dry oven (JSOF-050, JSR, South Korea) at $65{ }^{\circ} \mathrm{C}$ for $24 \mathrm{~h}$. Finally, the cured PDMS cap was detached from the mold and used to cover the plunger head. In the same manner, a PDMS cap for the support was also fabricated for use in the experiments.

Pressure Measurements of the PDV System. When the linear solenoid was actuated, the plunger struck the pressure cap placed on the sensor of the load cell. As described in the previous section, the mechanical force was converted to an electrical signal by the sensor of the load cell and displayed on an LCD. The load cell was only able to measure force in Newtons and consequently the measured force needed to be converted to units of pressure. The contact area was fixed to 2.0 $\times 10^{-5} \mathrm{~m}^{2}$ (5 mm diameter circle). Therefore, the load pressure was calculated by dividing the force with the area.

Paper-Based ELISA with PDVs. To construct a paperbased ELISA device with PDVs, four different functional membrane pads were prepared. A nitrocellulose paper was prepared by pipetting $0.2 \mu \mathrm{L}$ of capture antibody $(2.1 \mathrm{mg} / \mathrm{mL}$; goat anti-mouse IgG (Fc specific) antibody, Sigma-Aldrich, MI, USA) onto a site at $20 \mathrm{~mm}$ from the bottom of nitrocellulose paper (CNPF-SN12 $10 \mu \mathrm{m}$, MDI membrane, Ambala, India; 5 
A

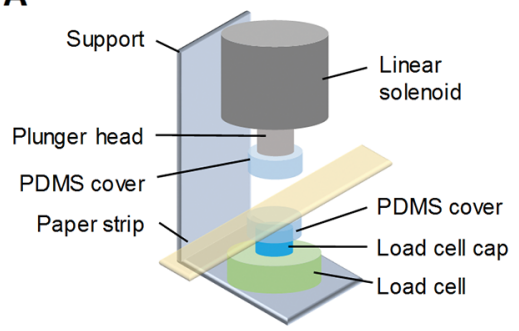

D

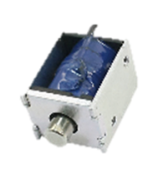

Linear solenoid
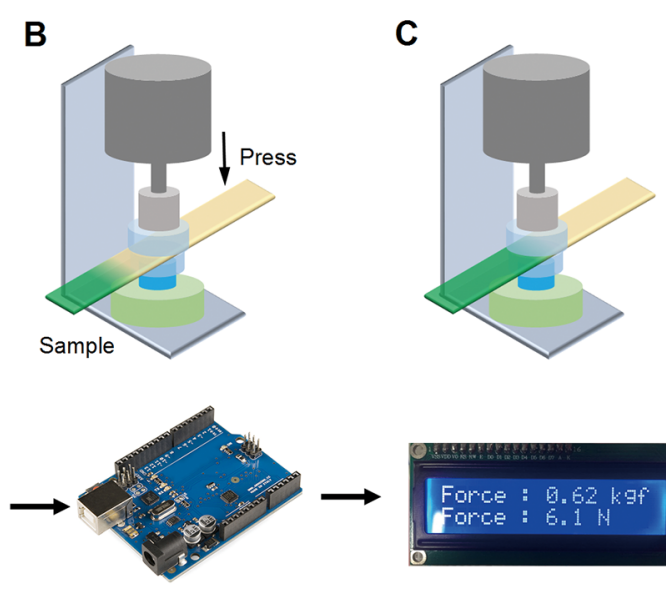

Microprocessor

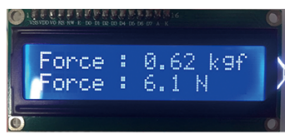

LCD

Figure 1. Operational steps of the proposed method. (A) Operation of the valve using the linear solenoid. A paper strip is placed beforehand onto the load cell cap capped with a PDMS cover. (B) Sample/reagent is dropped onto the inlet of a paper strip and the linear solenoid actuated. (C) Flow is then prohibited by the plunger head. (D) Configuration of the PDV system to measure mechanical pressure. When voltage is applied to the linear solenoid, the plunger presses the paper strip placed on the load cell, after which electrical signals are converted by the microprocessor and the force of the linear solenoid is displayed on the LCD.

$\mathrm{mm} \times 25 \mathrm{~mm})$ and dried for $10 \mathrm{~min}$ at room temperature. The nitrocellulose was blocked using 0.2\% (v/v) Tween 20 (P9416, Sigma-Aldrich) in $10 \mathrm{mM}$ phosphate buffered saline (PBS; LB004-02, Welgene, South Korea) with 5\% skimmed milk for $30 \mathrm{~min}$, after which the nitrocellulose paper was washed with $0.05 \%(\mathrm{v} / \mathrm{v})$ tween 20 in $10 \mathrm{mM}$ PBS for $30 \mathrm{~min}$ and dried for $1 \mathrm{~h}$ at $37{ }^{\circ} \mathrm{C}$. Then, $1 \mu \mathrm{L}$ of detection antibody $(100 \mu \mathrm{g} / \mathrm{mL}$; goat anti-mouse IgG-HRP (Fab specific) antibody, SigmaAldrich) in $10 \mathrm{mM}$ PBS containing $0.2 \%(\mathrm{v} / \mathrm{v})$ Tween 20 and $20 \%$ (w/v) sucrose (84100, Sigma-Aldrich) was immobilized onto a site at $5 \mathrm{~mm}$ from the bottom of the nitrocellulose paper and dried for $10 \mathrm{~min}$ at room temperature. Substrate channel (3 $\mathrm{mm} \times 15 \mathrm{~mm})$ and washing buffer channel $(3 \mathrm{~mm} \times 35 \mathrm{~mm})$ were prepared from chromatography grade 1 paper. The channels were then blocked with $0.2 \%(\mathrm{v} / \mathrm{v})$ Tween 20 in 10 mM PBS with 5\% BSA (A2153, Sigma-Aldrich) for $30 \mathrm{~min}$ and washed with the washing buffer for $30 \mathrm{~min}$ after which the channels were dried for $1 \mathrm{~h}$ at $37^{\circ} \mathrm{C}$. The nitrocellulose paper, sample pad (grade 319, Ahlstrom, Helsinki, Finland), channels for substrate and washing buffer, and absorbent pad (GB 005, GE Healthcare Whatman) were assembled on an adhesive plastic backing with an overlap of about $2 \mathrm{~mm}$.

Two reservoirs (15 $\mathrm{mm}$ diameter, $10 \mathrm{~mm}$ height) for the washing buffer and substrate $\left(3,3^{\prime}, 5,5^{\prime}\right.$-tetramethylbenzidine, TMB; T0565, Sigma-Aldrich) were fabricated by 3D printer and placed at the end of the washing and substrate channels. The two PDVs were positioned $10 \mathrm{~mm}$ from the end of the substrate and washing channels.

Image Analysis. At $15 \mathrm{~min}$ after opening valve 2, colorimetric intensity of the test zone was measured using a digital camera (NX1, Samsung Electronics, South Korea). Gray scale values of each detection zone were measured by image analysis software Image $\mathrm{J}$ and subtracted from the background signal.

\section{RESULTS AND DISCUSSION}

Operation of the PDV System. The PDV system was composed of a support, a linear push-pull solenoid with a plunger head, and a load cell with a cap to expand and flatten the contact area with the paper strips. The alignment between the plunger head and the load cell was critical to obtain accurate mechanical pressure. With the Arduino microprocessor, we were successfully able to control the integrated PDV system and visualize the mechanical force, which was converted to pressure (Figure 1). As a first step, we applied a voltage to the linear solenoid and confirmed that the plunger head was completely occluded by the load cell cap and pressed against the paper strip. The distance between the linear solenoid and the load cell cap affected the stroke distance of the plunger head. We used a linear solenoid with a $10 \mathrm{~mm}$ stroke and set the stroke as $0 \mathrm{~mm}$ when the plunger head was not moving. Results showed that the higher the voltage, the higher the pressure (Figure 2). While the pressure was almost constant

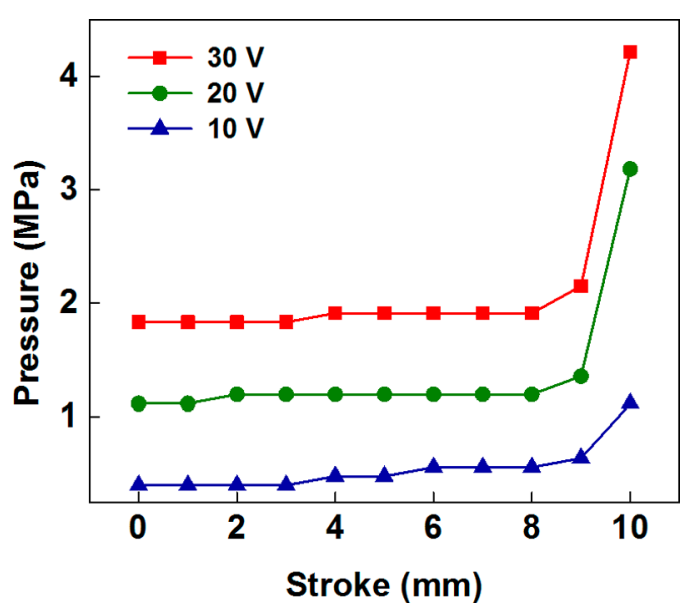

Figure 2. Pressure as a function of the stroke and the applied voltage. While the pressure was almost constant for strokes between 0 and 8 $\mathrm{mm}$, it significantly increased above the $9 \mathrm{~mm}$ stroke.

for strokes between 0 and $8 \mathrm{~mm}$, it significantly increased above a $9 \mathrm{~mm}$ stroke. Based on this result, we set the stroke distance as $9.5 \mathrm{~mm}$ to efficiently deliver pressure onto the paper strips.

Characterization for Fast and Reproducible Fluidic Control. The principal points in the development of in vitro diagnostic platforms are to make them fast and reproducible 
Filter paper

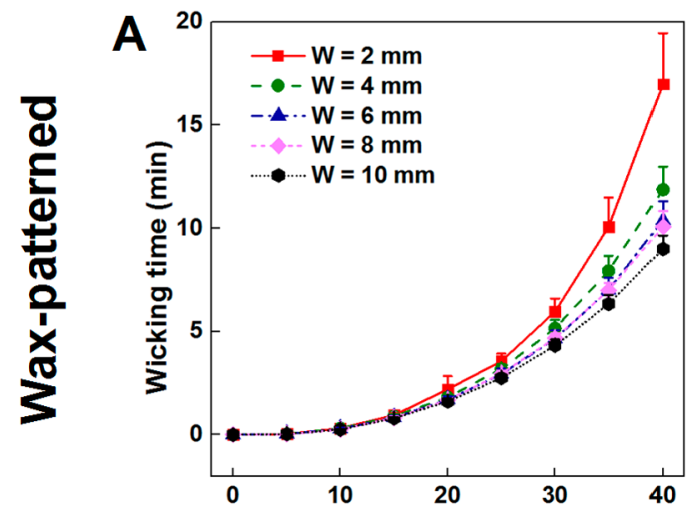

Distance from fluid source $(\mathrm{mm})$
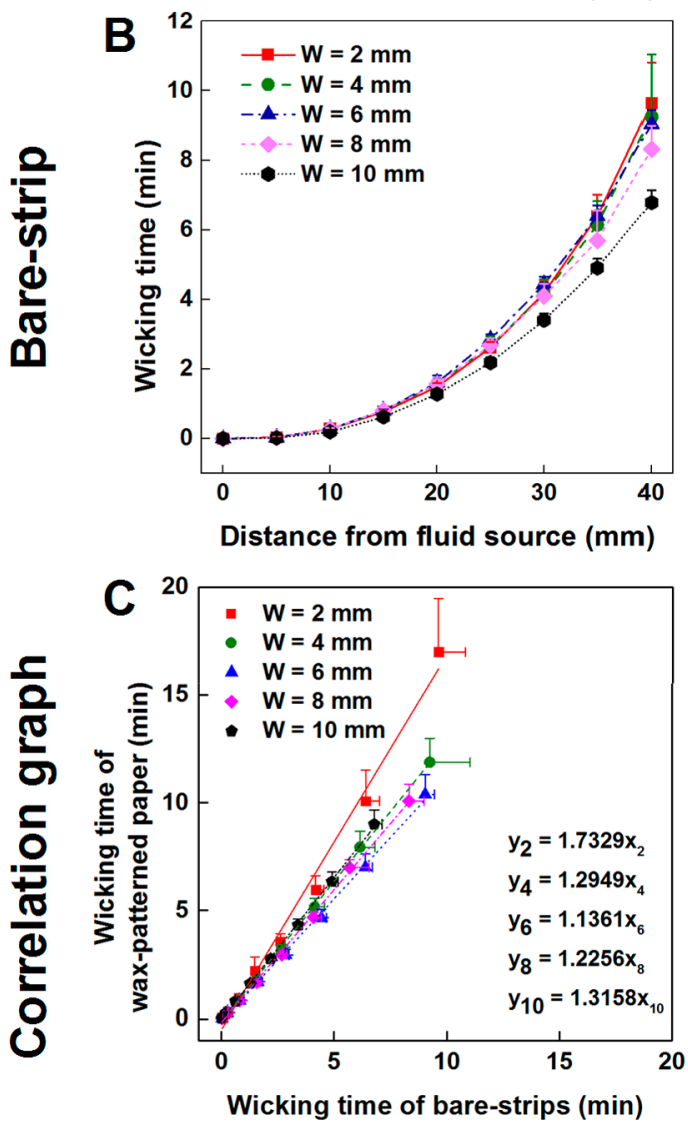

\section{Chromatography paper}

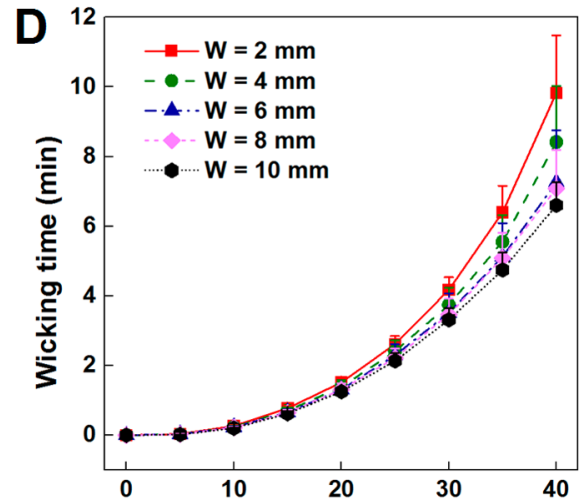

Distance from fluid source $(\mathrm{mm})$
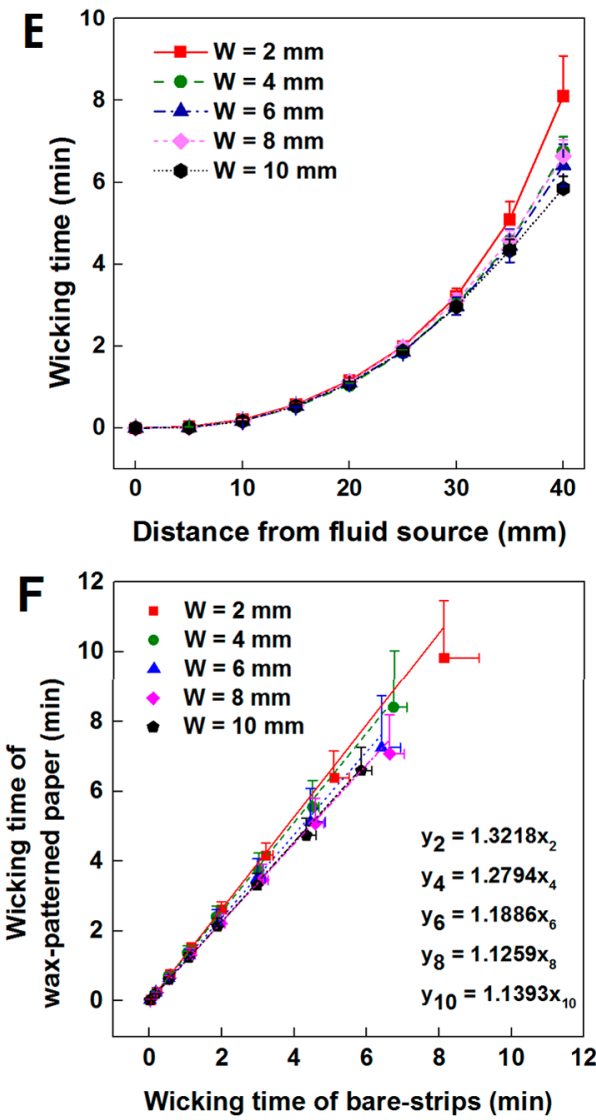

Figure 3. Wicking time for width-dependent wax-patterned/bare-strip channels made with chromatography/filter paper (40 mm length). (A) Wicking time results with wax-patterned filter channels. (B) Wicking time results with bare-strip filter channels. (C) Correlation graph of wicking time results between wax-patterned and bare-strip channels. (D) Wicking time results of chromatography with wax-patterned channels. (E) Wicking time results of chromatography with bare-strip channels. (F) Correlation graph of wicking time results between wax-patterned and bare-strip channels. The error bars represent the standard deviation of the experiments $(n=10)$.

with small sample volumes. Therefore, in order to find the optimal conditions satisfying the above three factors (speed, reproducibility, and sample usage), we checked the wicking time (the time taken by the fluid to flow from inlet to outlet) according to the grade of the paper, channel width, and fabrication method.

We selected two paper grades (chromatography grade 1 and filter grade 1) which have faster flow rates than other paper grades, such as grade 5 and 6 filter papers (see Figure $S 1$ in the Supporting Information). Differences in flow rate can be explained by the different pore size and porosities of the paper, as indicated by the Washburn equation and Darcy's law (see eqs 1 and 2 in the Supporting Information). Larger pore size and/or higher porosity result in higher flow rate. Since chromatography grade 1 paper has a higher porosity but the same size of pores, compared to grade 1 filter paper, it should display a higher flow rate.

The channel widths were chosen as $2,4,6,8$, and $10 \mathrm{~mm}$. We fabricated the paper channels with a typical strip-type form as well as by wax printing, which is a representative method for easy and fast fabrication. Each of the manufactured strips of papers was made with a length of $40 \mathrm{~mm}$ and soaked in a $2 \mathrm{~mL}$ 
green food dye solution. The wicking time was measured at intervals of $5 \mathrm{~mm}$ from the inlet (position, $0 \mathrm{~mm}$ ) to the outlet (position, $40 \mathrm{~mm}$ ) ports. As shown in Figure 3, faster flow was achieved with chromatography paper and this tendency was identical in both the bare-strip and wax-patterned channels. Panels $\mathrm{C}$ and $\mathrm{F}$ of Figure 3 show the correlation of wicking time between the bare-strip and the wax-patterned channels. Results indicate that the slopes of the graphs were greater than 1 for both filter and chromatography papers. Except for the case of 2 $\mathrm{mm}$ wax-patterned filter paper, the average slope converged to 1.2 in most cases, indicating that the flow velocity in the barestrip channels was 1.2-fold faster than that in the wax-patterned channels. Thus, the combination of bare-strip channel and grade 1 chromatography paper was superior in terms of flow velocity.

Moreover, we found that channel width was inversely proportional to wicking time (Figure 3A, B, D, E); i.e., the wider the channel, the faster the flow rate. This can be explained by the surface tension that occurs in the hydrophobic periphery, which acts in opposition to the flow. ${ }^{36}$ Although a wider channel exhibited faster flows, it was speculated that it would consume more reagents and samples. In terms of reproducibility, the bare-strip channel generally performs better than the wax-patterned channel. In particular, the bare-strip channel made of chromatography paper showed the most reliable reproducibility for all channel widths except $2 \mathrm{~mm}$ (Figure 3E). To summarize, after considering speed, reproducibility of fluidic transportation, and sample usage, we decided that the $4 \mathrm{~mm}$ wide bare-strip channel made of chromatography paper was optimal.

Bifunctional PDV System: Flow Velocity Control and Valve. Mechanical pressure is applied to the paper channel as the plunger head comes down and contacts the load cell cap. However, a cap made with a $3 \mathrm{D}$ printer might be rough and is not guaranteed to be sufficiently flat due to poor precision during fabrication. As the contact areas between the plunger head and the cap definitely affects the valve's efficiency, we fabricated PDMS covers and placed them under the plunger head and on the cap of the load cell in order to improve valve efficiency due to the elastic properties of PDMS. After that, the fluidic time delay was measured and compared between paper strips with and without the PDMS covers. The chromatographic paper strip was $40 \mathrm{~mm}$ long, and the plunger head was located in the $15-20 \mathrm{~mm}$ area away from the inlet. The time delay was calculated by subtracting the time it took for the fluid to reach the $25 \mathrm{~mm}$ point with no pressure during the corresponding time. Figure 4 shows the time delay depending on several pressures with and without PDMS covers. As expected, we confirmed that the time delay increased as the pressure became higher, while the PDMS covers significantly extended it. Notably, not only did the variation of the time delay decrease but also the relationship between the applied pressure and time delay became increasingly linear with the PDMS covers. Therefore, we were able to confirm that the PDMS covers improved the valve efficiency and reproducibility of fluidic control.

Based on the previous results, we tried to control the flow velocity with the PDV on paper. Figure 5A shows the flow velocity as a function of the pressure, covering the distance from the valve region to the outlet. The flow velocity was calculated by dividing the strip length of the strip between $L_{20}$ and $L_{40}(20 \mathrm{~mm})$ with the time it took for the dye to flow from $L_{20}$ to the end of the strip. Pressure was applied to the actuation

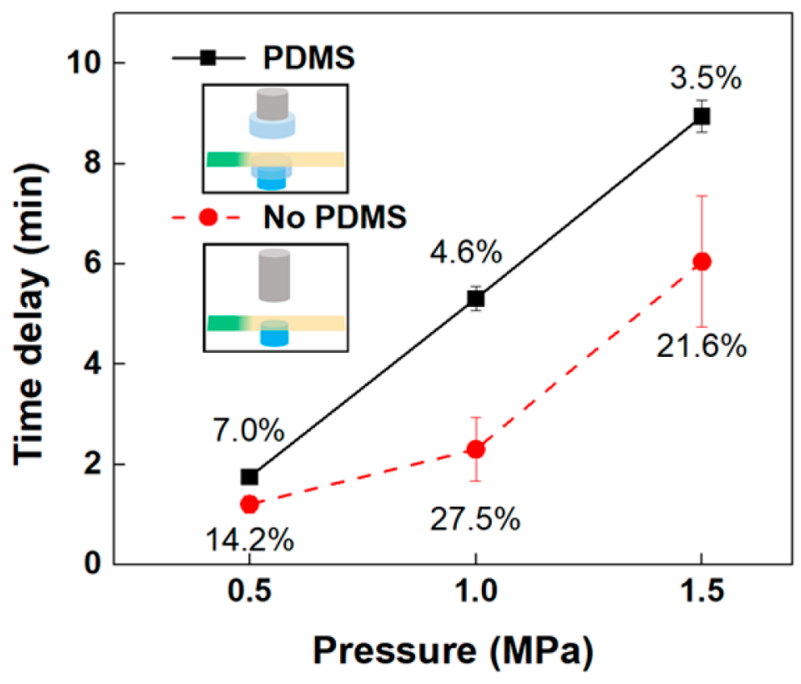

Figure 4. Time delay by the pressure driven active valve. The black solid line shows the time delay of the plunger head with the PDMS cover, while the red dashed line indicates the time delay with a bare plunger head. Percentage values represent the CV for each condition. The error bars represent the standard deviation of the experiment $(n=$ $3)$.

point $\left(L_{15}-L_{20}\right)$. The results show that the velocity linearly decreased when the pressure increased. We confirmed that the PDV system enables quantitative fluidic velocity control even on paper-based platforms.

Beyond the delay effect, various pressures were applied to observe the flow in order to confirm whether the platform could also function as an active valve. At up to $1.5 \mathrm{MPa}$, the flow was observed according to the degree of pressure. However, there was no flow in the actuation point from 2 $\mathrm{MPa}$ onward, thus confirming the function of the PDV as a valve (Figure 5B,C). Thereafter, when the valve was opened, the fluid flowed fairly well again and the slope of the fluidic flow was almost equivalent to that of the control.

If it is possible to open and close a single valve many times, we then envision that repetitive fluidic control can be performed on one type of reagent, such as multiple washing steps. This not only makes the device smaller by reducing the number of identical performing channels but also simplifies the POC paper device. As shown in Figure 5D, when the valve was repetitively closed and opened for four times there was an instant response of the flow, without delay. Moreover, the flow velocity was confirmed to be similar without reduced velocities as compared with the control. While most studies on paper valves have shown techniques to operate single-use valves, one approach demonstrated a reconfigurable valve allowing multiple actuation steps. ${ }^{35}$ However, there was a time delay in actuation of the wax valve. Moreover, there was concern about the effect of heating on heat-sensitive biological samples and reagents. To the best of our knowledge, our PDV system is the first demonstration that simultaneously achieves not only precise fluidic velocity control but also active valve actuation with programmability, repeatability, fast response, and compatibility with biological samples.

Paper-Based ELISA with PDVs. With the flow control ability of our PDV system, a multistep assay, such as ELISA, can be performed. As a proof-of-concept, we conducted HRP-based ELISA with mouse IgG as a target analyte. As shown in Figure $6 \mathrm{~A}$, there is a nitrocellulose paper for target detection in the 
A

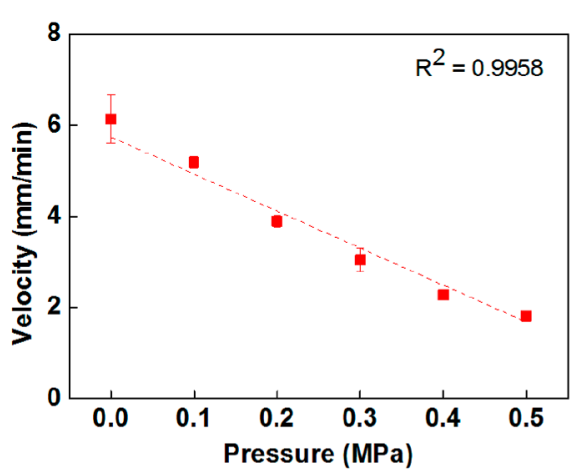

C

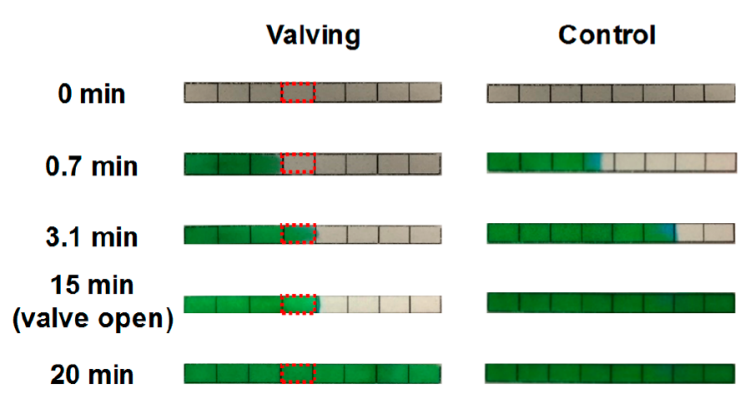

B

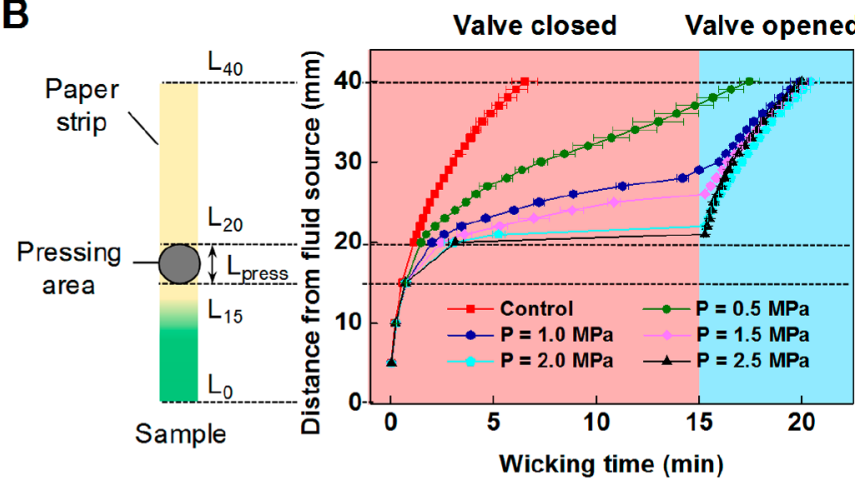

D

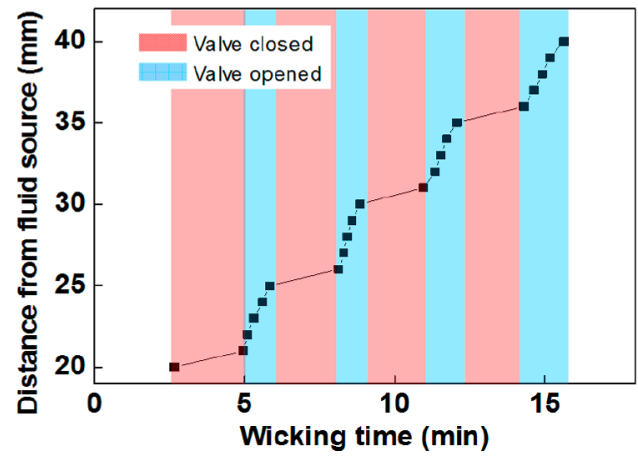

Figure 5. Bifunctional manipulation of flow velocity control and valve actuation based on a paper. (A) Flow results according to the pressure driven valve on strips when changing the pressure from 0 to $0.5 \mathrm{MPa}$. Pressure was applied to the actuation point $\left(L_{15}-L_{20}\right)$. (B) Flow results according to the pressure driven valve on strips when changing the pressure from 0 to $2.5 \mathrm{MPa}$. Pressure was applied to the actuation point $\left(L_{15}-L_{20}\right)$. The valve opened 15 min after valve actuation. The error bars represent the standard deviation of the experiment $(n=3)$. (C) Time-lapse images of flow on paper strips with $2.5 \mathrm{MPa}$ applied to the actuation point $\left(L_{15}-L_{20}\right)$ and without pressure (control). (D) Repeatability of the pressure driven valve. The flow instantly responded to the operation of the valve while the velocity did not significantly change with the number of valve operations.

center of the device. The black circle indicates the dried detection antibody (goat anti-mouse IgG-HRP). Capture antibody (goat anti-mouse $\operatorname{IgG}$ ) was immobilized onto a site $20 \mathrm{~mm}$ from bottom of the nitrocellulose paper (test zone). Furthermore, perpendicular to the nitrocellulose paper, there were two channels flanked with their respective 3D-printed reservoirs. A washing buffer was loaded in reservoir 1 , and substrate (TMB) for colorimetric reaction of HRP was loaded in reservoir 2. For the timed delivery of the washing buffer and the substrate, two PDVs $\left(V_{1}\right.$ and $\left.V_{2}\right)$ were placed on the respective channels.

With the PDVs closed, there was no flow off the reagents to the detection zone (Figure 6A). First, the sample (mouse IgG) solution was introduced on the sample pad and flowed toward the absorbent pad by means of capillary force (Figure 6B). In this process, mouse IgG interacted with detection antibody (black circle). Then, the antibody complex was bound to the capture antibody at the test zone. To wash off unbound detection antibody at the test zone, a washing step is essential. Therefore, valve $1\left(V_{1}\right)$ was opened for $15 \mathrm{~min}$ after sample introduction and excess detection antibodies were washed off (Figure $6 \mathrm{C})$. To visualize the results, valve $2\left(\mathrm{~V}_{2}\right)$ was opened for $15 \mathrm{~min}$ after washing for substrate delivery (Figure 6D). The HRP labeled detection antibodies reacted with the substrate complex for $15 \mathrm{~min}$ and produced a purple colored precipitant (Figure 6D; blue circle).

Figure $6 \mathrm{E}$ shows the results of paper-based ELISA at six different sample concentrations of mouse- $\operatorname{IgG}(0.05,0.1,0.25$, $0.5,0.75$, and $1 \mu \mathrm{g} / \mathrm{mL})$. All the colorimetric results were quantified and plotted (Figure 6F). With this proof-of-concept we have demonstrated that a PDV-based system can successfully perform a multistep analytical assay, such as a paper-based ELISA.

\section{CONCLUSIONS}

We have demonstrated a novel paper fluidic control system, enabling not only sophisticated flow velocity control but also active and repetitive valve actuation. The PDV presents many advantages, including programmability, reproducibility, fast response, and compatibility with biological samples, when compared to conventional devices. With its unique and versatile performance, we expect the PDV device to become an essential paper-based fluidic control unit for lateral flow devices used in point-of-care applications. We envision paper-based cartridges that can be slotted under the reservoirs containing washing and detection solution. Then only the sample is required to be manually loaded.

Although it might be pointed out that adding solenoid actuators will increase the cost of the lateral flow device, this can also be claimed for other active paper-based valves. In this case, not the cost of the paper device will increase but that of the control system. However, one should keep in mind that the costs of the reagents far outweigh those of the solenoid. Moreover, we believe that our PDV-based system will reduce reagent use, and thereby the cost of operation, justifying the initial cost of the control system. In addition, we believe that this approach has significant value in terms of versatility and the 

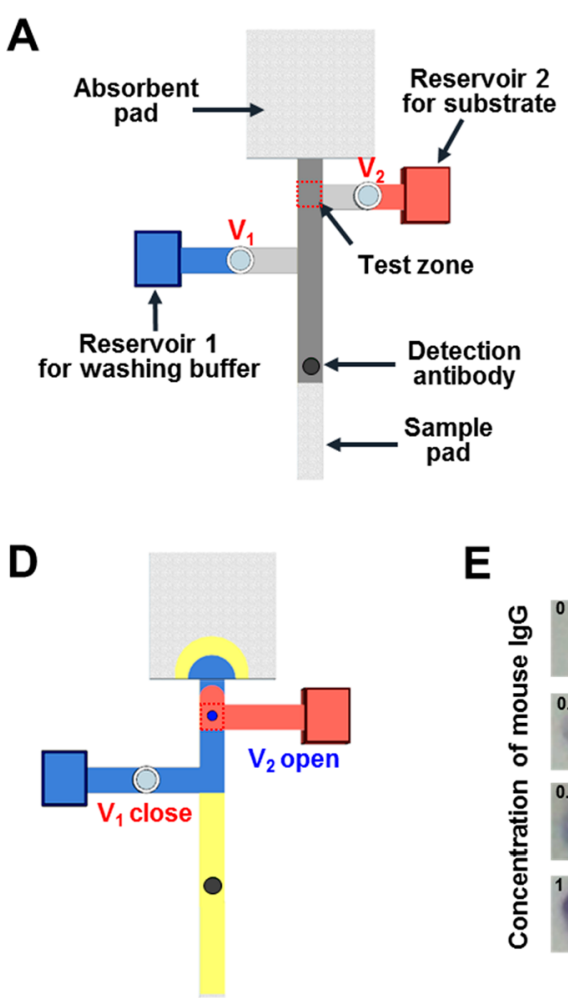

E
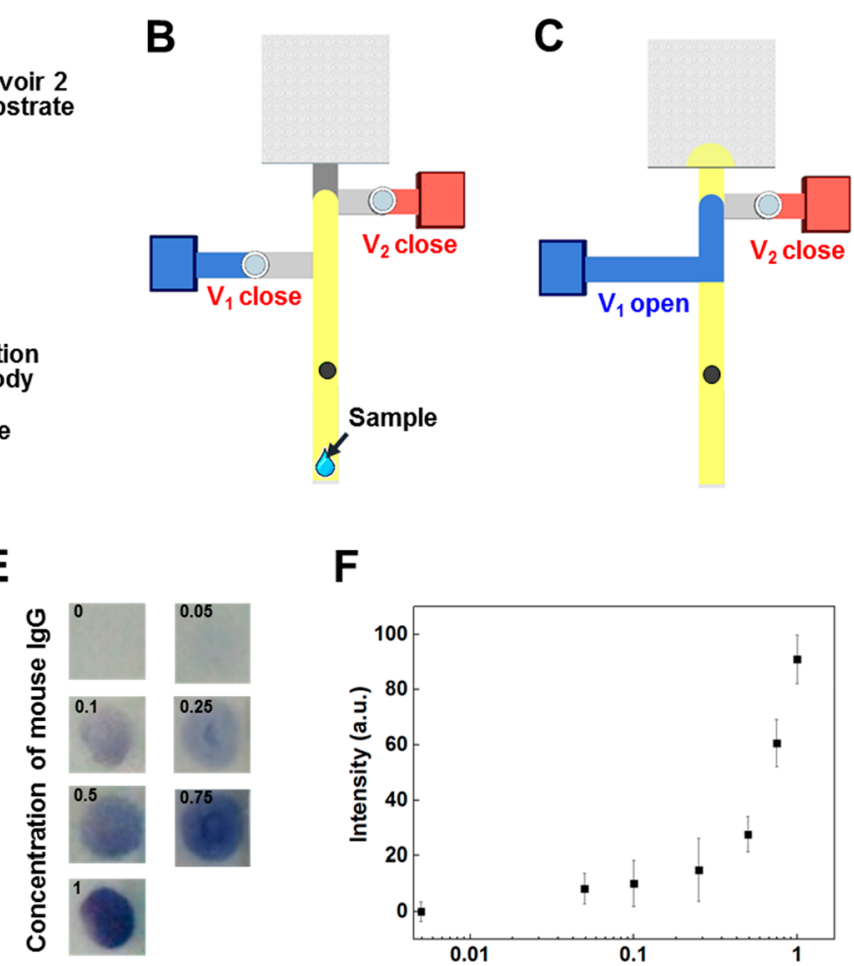

$\mathbf{F}$

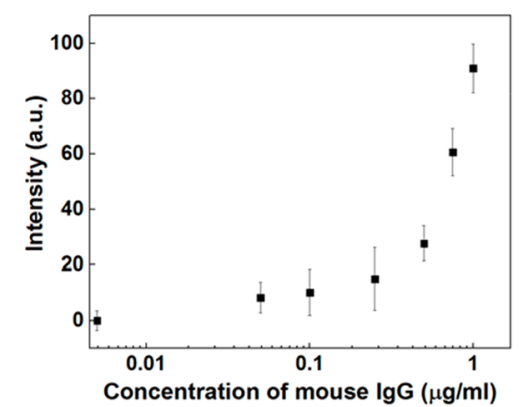

Figure 6. Paper-based ELISA with PDVs. (A) Schematic illustration of the PDV device applied to ELISA. The black circle on the nitrocellulose paper indicates immobilized detection antibody (goat anti-mouse IgG-HRP). Capture antibody (goat anti-mouse IgG) is immobilized onto the test zone (red dotted square). Two reservoirs and channels are placed next to the nitrocellulose paper for the delivery of washing buffer and substrate to the test zone, which are controlled by their respective PDVs $\left(V_{1}\right.$ and $\left.V_{2}\right)$. An absorbent pad is placed above the nitrocellulose paper facilitates the flow. (B) The test sample is loaded on the sample pad and moves to the test zone. (C) The washing step is commenced by opening $\mathrm{V}_{1}$. (D) By opening $\mathrm{V}_{2}$, HRP labeled detection antibody reacts with the substrate. The blue circle indicates the colorimetric result. (E) Images of the ELISA results using $0,0.05,0.1,0.25,0.5,0.75$, and $1 \mu \mathrm{g} / \mathrm{mL}$ mouse IgG as test sample. (F) Quantified colorimetric intensity results according to the concentration of mouse $\operatorname{IgG}(n=3)$.

ability to apply it to more complicated assays on a paper diagnostics platform.

\section{ASSOCIATED CONTENT}

\section{S Supporting Information}

The Supporting Information is available free of charge on the ACS Publications website at DOI: 10.1021/acs.analchem.7b03791.

Optimization of paper grades on flow velocity (PDF)

\section{AUTHOR INFORMATION}

\section{Corresponding Author}

*E-mail: kms@dgist.ac.kr. Tel.: +82-53-785-1740. Fax: +82-53785-1819.

\section{ORCID $\odot$}

Minseok S. Kim: 0000-0002-4268-6886

\section{Author Contributions}

${ }^{\perp}$ T.H.K. and Y.K.H. contributed equally to this work.

Notes

The authors declare no competing financial interest.

\section{ACKNOWLEDGMENTS}

This research was supported by the National Research Foundation of Korea (NRF; Grant Nos. NRF2017M3A9E9072667 and NRF-2015R1C1A1A01054292) funded by the Ministry of Science and ICT.

\section{REFERENCES}

(1) Martinez, A. W.; Phillips, S. T.; Whitesides, G. M.; Carrilho, E. Anal. Chem. 2010, 82, 3-10.

(2) Mahato, K.; Srivastava, A.; Chandra, P. Biosens. Bioelectron. 2017, 96, 246-259.

(3) Abe, K.; Suzuki, K.; Citterio, D. Anal. Chem. 2008, 80, 69286934.

(4) Martinez, A. W.; Phillips, S. T.; Butte, M. J.; Whitesides, G. M. Angew. Chem., Int. Ed. 2007, 46, 1318-1320.

(5) Dornelas, K. L.; Dossi, N.; Piccin, E. Anal. Chim. Acta 2015, 858, 82-90.

(6) Cai, L.; Wang, Y.; Wu, Y.; Xu, C.; Zhong, M.; Lai, H.; Huang, J. Analyst 2014, 139, 4593-4598.

(7) Noh, H.; Phillips, S. T. Anal. Chem. 2010, 82, 8071-8078.

(8) Noh, H.; Phillips, S. T. Anal. Chem. 2010, 82, 4181-4187.

(9) Siegel, A. C.; Phillips, S. T.; Wiley, B. J.; Whitesides, G. M. Lab Chip 2009, 9, 2775-2781.

(10) Li, M.; Tian, J.; Al-Tamimi, M.; Shen, W. Angew. Chem., Int. Ed. 2012, 51, 5497-5501.

(11) Cate, D. M.; Adkins, J. A.; Mettakoonpitak, J.; Henry, C. S. Anal. Chem. 2015, 87, 19-41.

(12) Nery, E. W.; Kubota, L. T. Anal. Bioanal. Chem. 2013, 405, $7573-7595$.

(13) Yetisen, A. K.; Akram, M. S.; Lowe, C. R. Lab Chip 2013, 13, 2210-2251.

(14) Ahmed, S.; Bui, M. P.; Abbas, A. Biosens. Bioelectron. 2016, 77, 249-263.

(15) Martinez, A. W.; Phillips, E. A.; Carrilho, E.; Thomas, S. W.; Sindi, H.; Whitesides, G. M. Anal. Chem. 2008, 80, 3699-3707. 
(16) Dungchai, W.; Chailapakul, O.; Henry, C. S. Anal. Chim. Acta 2010, 674, 227-233.

(17) Chen, X.; Chen, J.; Wang, F.; Xiang, X.; Luo, M.; Ji, X.; He, Z. Biosens. Bioelectron. 2012, 35, 363-368.

(18) Cheng, C. M.; Martinez, A. W.; Gong, J.; Mace, C. R.; Phillips, S. T.; Carrilho, E.; Mirica, K. A.; Whitesides, G. M. Angew. Chem., Int. Ed. 2010, 49, 4771-4774.

(19) Wang, S.; Ge, L.; Song, X.; Yu, J.; Ge, S.; Huang, J.; Zeng, F. Biosens. Bioelectron. 2012, 31, 212-218.

(20) Hu, J.; Wang, S.; Wang, L.; Li, F.; Pingguan-Murphy, B.; Lu, T. J.; Xu, F. Biosens. Bioelectron. 2014, 54, 585-597.

(21) Chen, H.; Cogswell, J.; Anagnostopoulos, C.; Faghri, M. Lab Chip 2012, 12, 2909-2913.

(22) Fridley, G. E.; Le, H.; Yager, P. Anal. Chem. 2014, 86, 64476453.

(23) Jahanshahi-Anbuhi, S.; Henry, A.; Leung, V.; Sicard, C.; Pennings, K.; Pelton, R.; Brennan, J. D.; Filipe, C. D. Lab Chip 2014, 14, 229-236.

(24) Li, X.; Tian, T. N.; Nguyen, T.; Shen, W. Anal. Chem. 2008, 80, 9131-9134.

(25) Li, X.; Zwanenburg, P.; Liu, X. Lab Chip 2013, 13, 2609-2614.

(26) Lutz, B. R.; Trinh, P.; Ball, C.; Fu, E.; Yager, P. Lab Chip 2011, 11, 4274-4278.

(27) Ouyang, Y.; Wang, S.; Li, J.; Riehl, P. S.; Begley, M.; Landers, J. P. Lab Chip 2013, 13, 1762-1771.

(28) Salentijn, G. I.; Hamidon, N. N.; Verpoorte, E. Lab Chip 2016, $16,1013-1021$.

(29) Martinez, A. W.; Phillips, S. T.; Nie, Z.; Cheng, C. M.; Carrilho, E.; Wiley, B. J.; Whitesides, G. M. Lab Chip 2010, 10, 2499-2504.

(30) Connelly, J. T.; Rolland, J. P.; Whitesides, G. M. Anal. Chem. 2015, 87, 7595-7601.

(31) Fu, E.; Liang, T.; Spicar-Mihalic, P.; Houghtaling, J.; Ramachandran, S.; Yager, P. Anal. Chem. 2012, 84, 4574-4579.

(32) Koo, C. K.; He, F.; Nugen, S. R. Analyst 2013, 138, 4998-5004.

(33) Toley, B. J.; Wang, J. A.; Gupta, M.; Buser, J. R.; Lafleur, L. K.; Lutz, B. R.; Fu, E.; Yager, P. Lab Chip 2015, 15, 1432-44.

(34) Houghtaling, J.; Liang, T.; Thiessen, G.; Fu, E. Anal. Chem. 2013, 85, 11201-11204.

(35) Phillips, E. A.; Shen, R.; Zhao, S.; Linnes, J. C. Lab Chip 2016, $16,4230-4236$.

(36) Walji, N.; MacDonald, B. Micromachines 2016, 7, 73. 